\title{
Trichotomy for Dynamical Systems in Banach Spaces
}

\author{
Codruţa Stoica \\ Department of Mathematics and Computer Science, Aurel Vlaicu University of Arad, 2 Elena Drăgoi Str, 310330 Arad, Romania
}

Correspondence should be addressed to Codruța Stoica; codruta.stoica@uav.ro

Received 25 July 2013; Accepted 13 August 2013

Academic Editors: G. Bonanno, F. Minhós, and G.-Q. Xu

Copyright (C) 2013 Codruța Stoica. This is an open access article distributed under the Creative Commons Attribution License, which permits unrestricted use, distribution, and reproduction in any medium, provided the original work is properly cited.

\begin{abstract}
We construct a framework for the study of dynamical systems that describe phenomena from physics and engineering in infinite dimensions and whose state evolution is set out by skew-evolution semiflows. Therefore, we introduce the concept of $\omega$-trichotomy. Characterizations in a uniform setting are proved, using techniques from the domain of nonautonomous evolution equations with unbounded coefficients, and connections with the classic notion of trichotomy are given. The statements are sustained by several examples.
\end{abstract}

\section{Introduction}

The possibility of reducing the nonautonomous case in the study of associated evolution operators to the autonomous case of evolution semigroups on various Banach function spaces can be considered as an important way towards applications issued from the real world. Of great importance in the study the solution of differential equations is the approach by evolution families, as the techniques from the domain of non autonomous equations with unbounded coefficients in infinite dimensions can be extended in this direction.

Appropriate for the study of evolution equations in infinite dimensions are also the skew-evolution semiflows, introduced by us in [1], as generalizations for evolution operators and skew-product semiflows, and whose applicability is pointed out, for example, by Bento and Silva in [2] and by Hai in $[3,4]$. Other asymptotic properties for skew-evolution semiflows are defined and characterized in $[5,6]$.

The techniques used in the investigation of exponential stability and exponential instability were generalized for the case of exponential dichotomy in $[7,8]$ and for the case of exponential trichotomy in $[9,10]$. The main idea in the study of trichotomy, introduced for the finite dimensional case by Sacker and Sell in [11], as a natural generalization of the concept of dichotomy is to obtain, at any moment, a decomposition of the state space into three subspaces: a stable subspace, an instable one, and a third one, the central manifold. The aim of the present study is to emphasize the property of $\omega$-trichotomy for skew-evolution semiflows in Banach spaces and to give several conditions in order to describe the behavior related to the third subspace.

\section{Skew-Evolution Semiflows}

Let $(X, d)$ be a metric space, $V$ a Banach space, and $V^{*}$ its topological dual. Let $\mathscr{B}(V)$ be the space of all $V$-valued bounded operators defined on $V$. The norm of vectors on $V$ and on $V^{*}$ and of operators on $\mathscr{B}(V)$ is denoted by $\|\cdot\|$. Let us consider that $Y=X \times V$ and $T=\left\{\left(t, t_{0}\right) \in \mathbb{R}_{+}^{2}: t \geq t_{0}\right\}$. $I$ is the identity operator.

Definition 1. A mapping $\varphi: T \times X \rightarrow X$ is called evolution semiflow on $X$ if the following properties are satisfied:

$$
\begin{aligned}
& \varphi(t, t, x)=x, \quad \forall(t, x) \in \mathbb{R}_{+} \times X, \quad\left(\mathrm{es}_{1}\right) \\
& \varphi\left(t, s, \varphi\left(s, t_{0}, x\right)\right)=\varphi\left(t, t_{0}, x\right), \quad \forall(t, s),\left(s, t_{0}\right) \in T, \\
& \forall x \in X .
\end{aligned}
$$

Definition 2. A mapping $\Phi: T \times X \rightarrow \mathscr{B}(V)$ is called evolution cocycle over an evolution semiflow $\varphi$ if it satisfies the following properties:

$$
\Phi(t, t, x)=I, \quad \forall t \geq 0, \forall x \in X, \quad\left(\mathrm{ec}_{1}\right)
$$




$$
\begin{aligned}
\Phi\left(t, s, \varphi\left(s, t_{0}, x\right)\right) \Phi\left(s, t_{0}, x\right) \\
\quad=\Phi\left(t, t_{0}, x\right), \quad \forall(t, s),\left(s, t_{0}\right) \in T, \forall x \in X .
\end{aligned}
$$

Definition 3. The mapping $C: T \times Y \rightarrow Y$ defined by

$$
C(t, s, x, v)=(\varphi(t, s, x), \Phi(t, s, x) v)
$$

where $\Phi$ is an evolution cocycle over an evolution semiflow $\varphi$, is called skew-evolution semiflow on $Y$.

Example 4. Let $f: \mathbb{R}_{+} \rightarrow \mathbb{R}_{+}^{*}$ be a decreasing function with the property that there exists $\lim _{t \rightarrow \infty} f(t)=a>0$. We denote by $\mathscr{C}=\mathscr{C}\left(\mathbb{R}_{+}, \mathbb{R}_{+}\right)$the set of all continuous functions $x$ : $\mathbb{R}_{+} \rightarrow \mathbb{R}_{+}$, endowed with the topology of uniform convergence on compact subsets of $\mathbb{R}_{+}$, metrizable by means of the distance

$$
\begin{gathered}
d(x, y)=\sum_{n=1}^{\infty} \frac{1}{2^{n}} \frac{d_{n}(x, y)}{1+d_{n}(x, y)}, \\
\text { where } d_{n}(x, y)=\sup _{t \in[0, n]}|x(t)-y(t)| .
\end{gathered}
$$

If $x \in \mathscr{C}$, then, for all $t \in \mathbb{R}_{+}$, we denote that $x_{t}(s)=x(t+s)$, $x_{t} \in \mathscr{C}$. Let $X$ be the closure in $\mathscr{C}$ of the set $\left\{f_{t}, t \in \mathbb{R}_{+}\right\}$. It follows that $(X, d)$ is a metric space, and the mapping $\varphi$ : $\Delta \times X \rightarrow X, \varphi(t, s, x)=x_{t-s}$ is an evolution semiflow on $X$.

We consider that $V=\mathbb{R}^{2}$, with the norm $\|v\|=\left|v_{1}\right|+\left|v_{2}\right|$, $v=\left(v_{1}, v_{2}\right) \in V$. If $u: \mathbb{R}_{+} \rightarrow \mathbb{R}_{+}^{*}$, then the mapping $\Phi_{u}:$ $\Delta \times X \rightarrow \mathscr{B}(V)$ defined by

$$
\Phi_{u}(t, s, x) v=\left(\frac{u(s)}{u(t)} e^{-\int_{s}^{t} x(\tau-s) d \tau} v_{1}, \frac{u(t)}{u(s)} e^{\int_{s}^{t} x(\tau-s) d \tau} v_{2}\right)
$$

is an evolution cocycle over $\varphi$, and $C=\left(\varphi, \Phi_{u}\right)$ is a skewevolution semiflow.

A particular class of skew-evolution semiflows is emphasized in the following.

Remark 5. Let us consider a skew-evolution semiflow $C=$ $(\varphi, \Phi)$ and a parameter $\lambda \in \mathbb{R}$. We define the mapping

$$
\Phi_{\lambda}: T \times X \longrightarrow \mathscr{B}(V), \quad \Phi_{\lambda}\left(t, t_{0}, x\right)=e^{-\lambda\left(t-t_{0}\right)} \Phi\left(t, t_{0}, x\right) .
$$

We observe that $C_{\lambda}=\left(\varphi, \Phi_{\lambda}\right)$ is also a skew-evolution semiflow, called $\lambda$-shifted skew-evolution semiflow on $Y$. We will call $\Phi_{\lambda}$ the $\lambda$-shifted evolution cocycle.

Example 6. Let $X$ be the metric space defined in the first Example. We define the mapping $\varphi_{0}: \mathbb{R}_{+} \times X \rightarrow X$, $\varphi_{0}(t, x)=x_{t}$, where $x_{t}(\tau)=x(t+\tau)$, for all $\tau \geq 0$, which is a classic semiflow on $X$. Let us consider for every $x \in X$ the parabolic system with Neumann's boundary conditions as follows:

$$
\begin{gathered}
\frac{\partial v}{\partial t}(t, y)=x(t) \frac{\partial^{2} v}{\partial y^{2}}(t, y), \quad t>0, \quad y \in(0,1) \\
v(0, y)=v_{0}(y), \quad y \in(0,1) \\
\frac{\partial v}{\partial y}(t, 0)=\frac{\partial v}{\partial y}(t, 1)=0, \quad t>0
\end{gathered}
$$

Let $V=\mathscr{L}^{2}(0,1)$ be a separable Hilbert space with the orthonormal basis $\left\{e_{n}\right\}_{n \in \mathbb{N}}, e_{0}=1, e_{n}(y)=\sqrt{2} \cos n \pi y$, where $y \in$ $(0,1), n \in \mathbb{N}$.

We denote that $D(A)=\left\{v \in \mathscr{L}^{2}(0,1), v(0)=v(1)=0\right\}$, and we define the operator

$$
A: D(A) \subset V \longrightarrow V, \quad A v=\frac{d^{2} v}{d y^{2}},
$$

which generates a $\mathscr{C}_{0}$-semigroup $S$, defined by

$$
S(t) v=\sum_{n=0}^{\infty} e^{-n^{2} \pi^{2} t}\left\langle v, e_{n}\right\rangle e_{n}
$$

where $\langle\cdot, \cdot\rangle$ denotes the scalar product in $V$. For every $x \in X$, let us define an operator $A(x): D(A) \subset V \rightarrow V, A(x)=$ $x(0) A$, which allows us to rewrite system (5) in $V$ as

$$
\begin{gathered}
\dot{v}(t)=A\left(\varphi_{0}(t, x)\right) v(t), \quad t>0, \\
v(0)=v_{0} .
\end{gathered}
$$

The mapping

$$
\Phi_{0}: \mathbb{R}_{+} \times X \longrightarrow \mathscr{B}(V), \quad \Phi_{0}(t, x) v=S\left(\int_{0}^{t} x(s) d s\right) v
$$

is a classic cocycle over the semiflow $\varphi_{0}$, and $C_{0}=\left(\varphi_{0}, \Phi_{0}\right)$ is a linear skew-product semiflow strongly continuous on $Y$. Also, for all $v_{0} \in D(A)$, we have obtained that $v(t)=$ $\Phi(t, x) x_{0}, t \geq 0$, is a strongly solution of system (8). As $C_{0}=\left(\varphi_{0}, \Phi_{0}\right)$ is a skew-product semiflow on $Y$, then the mapping $C: T \times Y \rightarrow Y, C(t, s, x, v)=(\varphi(t, s, x), \Phi(t, s, x) v)$, where

$$
\begin{array}{r}
\varphi(t, s, x)=\varphi_{0}(t-s, x), \quad \Phi(t, s, x)=\Phi_{0}(t-s, x), \\
\forall(t, s, x) \in T \times X,
\end{array}
$$

is a skew-evolution semiflow on $Y$. Hence, the skew-evolution semiflows generalize the notion of skew-product semiflows.

More directly, if $\Phi(t, s, x)$ is the solution of the Cauchy problem

$$
\begin{gathered}
v^{\prime}(t)=A(\varphi(t, s, x)) v(t), \quad t>s, \\
v(s)=x
\end{gathered}
$$

then $C=(\varphi, \Phi)$ is a linear skew-evolution semiflow.

Let us recall the definition of a semigroup of linear operators, and let us give an example which shows that this is generating a skew-evolution semiflow. 
Definition 7. A mapping $S: \mathbb{R}_{+} \rightarrow \mathscr{B}(V)$ is called semigroup of linear operators on $V$ if the following relations hold:

$$
\begin{gathered}
S(0)=I, \\
S(t) S(s)=S(t+s), \quad \forall(t, s) \in \mathbb{R}_{+}^{2} .
\end{gathered}
$$

Example 8. One can naturally associate to every semigroup of operators the mapping $\Phi_{S}: T \times X \rightarrow \mathscr{B}(V)$, defined by $\Phi_{S}(t, s, x)=S(t-s)$, which is an evolution cocycle on $V$ over evolution semiflows given, for example, by $\varphi(t, s, x)=x_{t-s}$ (see Example 4).

Other examples of skew-evolution semiflows are given in [6]. The asymptotic properties, as well as their characterizations, are given by means of norms of the trajectories or orbits of $v$, given by $t \rightarrow \Phi\left(t, t_{0}, x\right) v$, which are considered measurable. A particular case of skew-evolution semiflows is given by the following.

Definition 9. A skew-evolution semiflow $C=(\varphi, \Phi)$ is *strongly measurable if, for every $\left(t, t_{0}, x, v^{*}\right) \in T \times X \times V^{*}$, the mapping given by $s \mapsto\left\|\Phi\left(t, s, \varphi\left(s, t_{0}, x\right)\right)^{*} v^{*}\right\|$ is measurable on $\left[t_{0}, t\right]$.

\section{On Trichotomy Issues}

We intend to give a new approach for the property of trichotomy for skew-evolution semiflows, the $\omega$-trichotomy. Some examples and connections with the classic concept of exponential trichotomy are also provided.

Let $C: T \times Y \rightarrow Y, C(t, s, x, v)=(\varphi(t, s, x), \Phi(t, s, x) v)$ be a skew-evolution semiflow on $Y$. We recall that a mapping $P: X \rightarrow \mathscr{B}(V)$ with the property

$$
P(x)^{2}=P(x), \quad \forall x \in X
$$

is called projections family on $V$.

Definition 10. A projections family $P: X \rightarrow \mathscr{B}(V)$ is said to be invariant relative to the skew-evolution semiflow $C=$ $(\varphi, \Phi)$ if

$$
\begin{array}{r}
\Phi(t, s, x) P(x)=P(\varphi(t, s, x)) \Phi(t, s, x), \\
\forall(t, s, x) \in T \times X .
\end{array}
$$

The splitting of the state space into three subspaces will be assured by the following.

Definition 11. Three projections families $\left\{P_{k}\right\}_{k \in\{1,2,3\}}$ are said to be compatible with a skew-evolution semiflow $C=(\varphi, \Phi)$ if

$\left(c_{1}\right)$ each of $P_{k}, k \in\{1,2,3\}$ is invariant relative to $C$;

$\left(c_{2}\right)$ for all $x \in X$, the projections verify the relations

$$
\begin{array}{r}
P_{1}(x)+P_{2}(x)+P_{3}(x)=I, \quad P_{i}(x) P_{j}(x)=0, \\
\forall i, j \in\{1,2,3\} \quad i \neq j .
\end{array}
$$

In what follows we introduce the elements which will allow us to introduce a new concept of trichotomy for skewevolution semiflows. We consider a mapping $\psi: \mathbb{R}_{+} \rightarrow \mathbb{R}_{+}^{*}$, and we define

$$
\begin{gathered}
A_{\psi}=\left\{\omega \in \mathbb{R}, \sup _{t \geq 0} \psi(t) e^{-\omega t}<\infty\right\}, \\
B_{\psi}=\left\{\omega \in \mathbb{R}, \inf _{t \geq 0} \psi(t) e^{-\omega t}>0\right\} .
\end{gathered}
$$

Remark 12 . For every function $\psi: \mathbb{R}_{+} \rightarrow \mathbb{R}_{+}^{*}$, the following statements hold:

(i) $A_{\psi} \neq \emptyset$ and $B_{\psi} \neq \emptyset$;

(ii) $\mu \in A_{\psi}$ implies the existence of a constant $M \in \mathbb{R}_{+}$ such that

$$
\psi(t) \leq M e^{\mu t}, \quad \forall t \geq 0
$$

(iii) $\lambda \in B_{h}$ implies that there exists a constant $m \in \mathbb{R}_{+}$ such that

$$
\psi(t) \geq m e^{\lambda t}, \quad \forall t \geq 0 .
$$

Let us denote that

$$
\begin{aligned}
& \bar{\omega}_{\psi}= \begin{cases}\inf A_{\psi}, & \text { if } A_{\psi} \neq \emptyset, \\
\infty, & \text { if } A_{\psi}=\emptyset,\end{cases} \\
& \underline{\omega}_{\psi}= \begin{cases}\sup B_{\psi}, & \text { if } B_{\psi} \neq \emptyset, \\
-\infty, & \text { if } B_{\psi}=\emptyset .\end{cases}
\end{aligned}
$$

Definition 13. A skew-evolution semiflow $C=(\varphi, \Phi)$ is called $\omega$-trichotomic if there exist three projections families $\left\{P_{k}\right\}_{k \in\{1,2,3\}}$ compatible with $C$ and some functions $\psi, \zeta, \chi$ : $\mathbb{R}_{+} \rightarrow \mathbb{R}_{+}^{*}$ with the properties

$$
\bar{\omega}_{\psi}<0, \quad \underline{\omega}_{\zeta}>0, \quad \bar{\omega}_{\chi}<\infty, \quad \underline{\omega}_{\chi}<0,
$$

such that

$$
\begin{aligned}
& \left(t_{1}\right)\left\|\Phi\left(t, t_{0}, x\right) P_{1}(x) v\right\| \leq \psi\left(t-t_{0}\right)\left\|P_{1}(x) v\right\| ; \\
& \left(t_{2}\right) \zeta\left(t-t_{0}\right)\left\|P_{2}(x) v\right\| \leq\left\|\Phi\left(t, t_{0}, x\right) P_{2}(x) v\right\| ; \\
& \left(t_{3}\right)\left\|\Phi\left(t, t_{0}, x\right) P_{3}(x) v\right\| \leq \chi\left(t-t_{0}\right)\left\|P_{3}(x) v\right\| \text { and } \chi(t- \\
& \left.\quad t_{0}\right)\left\|\Phi\left(t, t_{0}, x\right) P_{3}(x) v\right\| \geq\left\|P_{3}(x) v\right\|, \\
& \quad \text { for all }\left(t, t_{0}\right) \in T \text { and all }(x, v) \in Y .
\end{aligned}
$$

Example 14. Let $f: \mathbb{R}_{+} \rightarrow(0, \infty)$ be a decreasing function with the property that there exists $\lim _{t \rightarrow \infty} f(t)=l>0$. Let us denote that $\lambda>f(0)$. Let us consider the Banach space $V=\mathbb{R}^{3}$ with the norm $\left\|\left(v_{1}, v_{2}, v_{3}\right)\right\|=\left|v_{1}\right|+\left|v_{2}\right|+\left|v_{3}\right|, v=$ $\left(v_{1}, v_{2}, v_{3}\right) \in V$. The mapping

$$
\begin{gathered}
\Phi: T \times X \longrightarrow \mathscr{B}(V), \\
\Phi\left(t, t_{0}, x\right) v \\
=\left(e^{-\lambda\left(t-t_{0}\right)+\int_{t_{0}}^{t} x\left(\tau-t_{0}\right) d \tau} v_{1}, e^{\int_{t_{0}}^{t} x\left(\tau-t_{0}\right) d \tau} v_{2},\right. \\
\left.e^{-\left(t-t_{0}\right) x(0)+\int_{t_{0}}^{t} x\left(\tau-t_{0}\right) d \tau} v_{3}\right),
\end{gathered}
$$


where $t \geq t_{0} \geq 0,(x, v) \in Y$, is an evolution cocycle over the evolution semiflow given in Example 4. We define the projections families $P_{1}, P_{2}, P_{3}: X \rightarrow \mathscr{B}\left(\mathbb{R}^{3}\right)$ by $P_{1}(x) v=\left(v_{1}\right.$, $0,0), P_{2}(x) v=\left(0, v_{2}, 0\right), P_{3}(x) v=\left(0,0, v_{3}\right)$, for all $x \in X$ and all $v=\left(v_{1}, v_{2}, v_{3}\right) \in \mathbb{R}^{3}$. The following inequalities

$$
\begin{aligned}
& \left\|\Phi\left(t, t_{0}, x\right) P_{1}(x) v\right\| \\
& \quad \leq e^{[-\lambda+x(0)](t-s)}\left\|\Phi\left(s, t_{0}, x\right) P_{1}(x) v\right\|, \\
& \left\|\Phi\left(t, t_{0}, x\right) P_{2}(x) v\right\| \\
& \quad \geq e^{l(t-s)}\left\|\Phi\left(s, t_{0}, x\right) P_{2}(x) v\right\|, \\
& \left\|\Phi\left(t, t_{0}, x\right) P_{3}(x) v\right\| \\
& \quad \leq e^{-x(0)(t-s)}\left\|\Phi\left(s, t_{0}, x\right) P_{3}(x) v\right\|, \\
& \left\|\Phi\left(t, t_{0}, x\right) P_{3}(x) v\right\| \\
& \quad \geq e^{-x(0)(t-s)}\left\|\Phi\left(s, t_{0}, x\right) P_{3}(x) v\right\|
\end{aligned}
$$

hold for all $(t, s),\left(s, t_{0}\right) \in T$ and all $(x, v) \in Y$. The mappings $\psi, \zeta, \chi: \mathbb{R}_{+} \rightarrow \mathbb{R}_{+}^{*}$, defined by

$$
\psi(u)=e^{[-\lambda+x(0)] u}, \quad \zeta(u)=e^{l u}, \quad \chi(u)=e^{-x(0) u}
$$

satisfy relations (19). For $s=t_{0}$ and according to Definition 2, $\left(\right.$ et $\left._{1}\right)$ we obtain relations (i)-(iii) in Definition 13 . Hence, $C$ is $\omega$-trichotomic.

Remark 15. For $P_{3}=0$, the property of $\omega$-dichotomy is obtained. If we consider $P_{2}=P_{3}=0$, we obtain the property of $\omega$-stability and for $P_{1}=P_{3}=0$, the property of $\omega$ instability.

In what follows, if $P_{k}$ is a given projections family, we will denote that

$$
\Phi_{k}(t, s, x)=\Phi(t, s, x) P_{k}(x),
$$

for every $(t, s),\left(s, t_{0}\right) \in T$ and $x \in X$. We remark that the following relations hold:

(i) $\Phi_{k}(t, t, x)=P_{k}(x), \forall(t, x) \in \mathbb{R}_{+} \times X$;

(ii) $\Phi_{k}\left(t, s, \varphi\left(s, t_{0}, x\right)\right) \Phi_{k}\left(s, t_{0}, x\right)=\Phi_{k}\left(t, t_{0}, x\right), \forall(t, s)$, $\left(s, t_{0}\right) \in T, \forall x \in X$.

Proposition 16. A skew-evolution semiflow $C=(\varphi, \Phi)$ is $\omega$ trichotomic if and only if there exist some constants $N_{1}, N_{2}$, $N_{3} \geq 1, v_{1}, v_{2}, v_{3}>0$ and three projections families $\left\{P_{k}\right\}_{k \in\{1,2,3\}}$ compatible with $C$ such that

$$
\begin{gathered}
e^{\nu_{1}\left(t-t_{0}\right)}\left\|\Phi_{1}\left(t, t_{0}, x\right) v\right\| \leq N_{1}\left\|P_{1}(x) v\right\|, \quad\left(\text { et }_{1}\right) \\
e^{\nu_{2}\left(t-t_{0}\right)}\left\|P_{2}(x) v\right\| \leq N_{2}\left\|\Phi_{2}\left(t, t_{0}, x\right) v\right\|, \quad\left(\mathrm{et}_{2}\right) \\
\left\|P_{3}(x) v\right\| \leq N_{3} e^{\nu_{3}\left(t-t_{0}\right)}\left\|\Phi_{3}\left(t, t_{0}, x\right) v\right\| \\
\leq N_{3}^{2} e^{2 v_{3}\left(t-t_{0}\right)}\left\|P_{3}(x) v\right\|,
\end{gathered}
$$

for all $\left(t, t_{0}\right) \in T$ and all $(x, v) \in Y$.
Proof. Necessity. Let $C$ be $\omega$-trichotomic. Then there exist three projections families compatible with $C$ and three functions $\psi, \zeta, \chi: \mathbb{R}_{+} \rightarrow \mathbb{R}_{+}^{*}$ with the properties

$$
\bar{\omega}_{\psi}<0, \quad \underline{\omega}_{\zeta}>0, \quad \bar{\omega}_{\chi}<\infty, \quad \underline{\omega}_{\chi}<0,
$$

such that relations (i)-(iii) of Definition 13 are verified. For $\mu \in A_{\psi}$, there exists a constant $M \in \mathbb{R}_{+}$such that $\psi(t) \leq$ $M e^{\mu t}$, for all $t \geq 0$, and for $\lambda \in B_{\psi}$ there exists a constant $m \in \mathbb{R}_{+}$with the property $\psi(t) \geq m e^{\lambda t}$, for all $t \geq 0$.

As $\bar{\omega}_{\psi}<0$, there exist $N_{1} \geq 1$ and $\nu_{1}>0$, such that $\psi(t) \leq N_{1} e^{-v_{1} t}$, for all $t \geq 0$. Hence, relation $\left(\mathrm{et}_{1}\right)$ is obtained. As $\underline{\omega}_{\zeta}>0$, it follows that there exist $N_{2} \geq 1$ and $\nu_{2}>0$, such that $\zeta(t) \geq N_{2} e^{\nu_{2} t}$, for all $t \geq 0$, which implies (et $\left.{ }_{2}\right)$. From $\bar{\omega}_{\chi}<\infty$ and $\underline{\omega}_{\chi}<0$, it follows that there exist $N_{3} \geq 1, \nu_{3}>0$ with the property

$$
\frac{1}{N_{3}} e^{-\gamma_{3} t} \leq \chi(t) \leq N_{3} e^{\nu_{3} t}, \quad \forall t \geq 0
$$

Hence, relation $\left(\mathrm{et}_{3}\right)$ is satisfied.

Sufficiency. Let us assume that there exist three projections families compatible with $C$ and $N_{1}, N_{2}, N_{3} \geq 1, v_{1}, v_{2}$, $v_{3}>0$ such that relations $\left(\mathrm{et}_{1}\right)-\left(\mathrm{et}_{3}\right)$ hold. Let us define the mappings $\psi, \zeta, \chi: \mathbb{R}_{+} \rightarrow \mathbb{R}_{+}^{*}$ by

$$
\psi(t)=N_{1} e^{-\nu_{1} t}, \quad \zeta(t)=N_{2}^{-1} e^{\nu_{2} t}, \quad \chi(t)=N_{3} e^{\nu_{3} t} .
$$

We obtain the relations

$$
\bar{\omega}_{\psi}<0, \quad \underline{\omega}_{\zeta}>0, \quad \bar{\omega}_{\chi}<\infty, \quad \underline{\omega}_{\chi}<0
$$

Hence, relations (i)-(iii) of Definition 13 are verified, and, therefore, $C$ is $\omega$-trichotomic, which ends the proof.

Remark 17. Proposition 16 is in fact the classic definition of exponential trichotomy. On the other hand, in Definition 13, the exponentials are not implied.

\section{Main Results}

We obtain a characterization for the property of trichotomy, by means of the shifted skew-evolution semiflow.

Theorem 18. A skew-evolution semiflow $C=(\varphi, \Phi)$ is $\omega$ trichotomic if and only if there exist three projections families $\left\{P_{k}\right\}_{k \in\{1,2,3\}}$ compatible with $C$ and

(i) the evolution cocycle $\Phi_{1}$ is exponentially stable;

(ii) the evolution cocycle $\Phi_{2}$ is exponentially instable;

(iii) there exists a constant $\lambda>0$ such that the $\lambda$-shifted evolution cocycle $\Phi_{\lambda}^{3}$ is exponentially stable and the $-\lambda$ shifted evolution cocycle $\Phi_{-\lambda}^{3}$ is exponentially instable. 
Proof. Necessity. Statements (i) and (ii) are obtained immediately from the necessity of Proposition 16. According to $\left(\mathrm{et}_{3}\right)$, there exist $N_{3} \geq 1$ and $\nu_{3}>0$ such that

$$
\begin{array}{r}
\left\|\Phi_{3}\left(t, t_{0}, x\right) v\right\| \leq N_{3} e^{v_{3}\left(t-t_{0}\right)}\left\|P_{3}(x) v\right\|, \\
\forall\left(t, t_{0}\right) \in T, \quad \forall(x, v) \in Y .
\end{array}
$$

Let us consider that $\lambda=2 \nu_{3}>0$. We obtain successively

$$
\begin{aligned}
\left\|\Phi_{\lambda}^{3}\left(t, t_{0}, x\right) v\right\| & =e^{-\lambda\left(t-t_{0}\right)}\left\|\Phi_{3}\left(t, t_{0}, x\right) v\right\| \\
& \leq N_{3} e^{-\lambda\left(t-t_{0}\right)} e^{\nu_{3}\left(t-t_{0}\right)}\left\|P_{3}(x) v\right\| \\
& =N_{3} e^{-\gamma_{3}\left(t-t_{0}\right)}\left\|P_{3}(x) v\right\|,
\end{aligned}
$$

for all $\left(t, t_{0}\right) \in T$ and all $(x, v) \in Y$, which shows that $\Phi_{\lambda}^{3}$ is exponentially stable. Also, we have

$$
\begin{array}{r}
e^{-v_{3}\left(t-t_{0}\right)}\left\|P_{3}(x) v\right\| \leq N_{3}\left\|\Phi_{3}\left(t, t_{0}, x\right) v\right\|, \\
\forall\left(t, t_{0}\right) \in T, \quad \forall(x, v) \in Y .
\end{array}
$$

If we consider that $\lambda=2 \nu_{3}>0$, we obtain, according also to Definition $2\left(\mathrm{ec}_{2}\right)$,

$$
\begin{aligned}
\left\|\Phi_{-\lambda}^{3}\left(s, t_{0}, x\right) v\right\| & =e^{\lambda\left(s-t_{0}\right)}\left\|\Phi_{3}\left(s, t_{0}, x\right) v\right\| \\
& \leq N_{3} e^{\nu_{3}(t-s)} e^{\lambda\left(s-t_{0}\right)}\left\|\Phi_{3}\left(t, t_{0}, x\right) v\right\| \\
& =N_{3} e^{-v_{3}(t-s)}\left\|\Phi_{-\lambda}^{3}\left(t, t_{0}, x\right) v\right\|,
\end{aligned}
$$

for all $(t, s),\left(s, t_{0}\right) \in T$ and all $(x, v) \in Y$, which proves that $\Phi_{-\lambda}^{3}$ is exponentially instable.

Sufficiency. Relations (i) and (ii) in Definition 13 are obtained from the sufficiency of Proposition 16. As there exists a constant $\lambda>0$ such that the skew-evolution semiflow $\Phi_{\lambda}^{3}$ is exponentially stable, then there exists some constants $N \geq 1$ and $v>0$ such that

$$
\begin{array}{r}
\left\|\Phi_{\lambda}^{3}\left(t, t_{0}, x\right) v\right\| \leq N e^{-v\left(t-t_{0}\right)}\left\|P_{3}(x) v\right\|, \\
\forall t \geq t_{0} \geq 0, \forall(x, v) \in Y .
\end{array}
$$

Further, we obtain

$$
\begin{aligned}
\left\|\Phi_{3}\left(t, t_{0}, x\right) v\right\| & =e^{\lambda\left(t-t_{0}\right)}\left\|\Phi_{\lambda}^{3}\left(t, t_{0}, x\right) v\right\| \\
& \leq N e^{\lambda\left(t-t_{0}\right)} e^{-v(t-s)}\left\|\Phi_{\lambda}^{3}\left(s, t_{0}, x\right) v\right\| \\
& =N e^{\lambda(t-s)} e^{-v(t-s)}\left\|\Phi_{3}\left(s, t_{0}, x\right) v\right\|,
\end{aligned}
$$

for all $(t, s),\left(s, t_{0}\right) \in T$ and all $(x, v) \in Y$. Denoting

$$
v_{3}= \begin{cases}\lambda-v, & \text { if } \lambda>v \\ 1, & \text { if } \lambda \leq v\end{cases}
$$

the first relation in $\left(\mathrm{et}_{3}\right)$ is obtained.
If there exists a constant $\lambda>0$ such that the $-\lambda$-shifted skew-evolution semiflow $C_{-\lambda}$ is exponentially instable, then there exist some constants $N \geq 1$ and $v>0$ such that

$$
\begin{aligned}
\left\|\Phi\left(s, t_{0}, x\right) v\right\| & =e^{-\lambda\left(s-t_{0}\right)}\left\|\Phi_{-\lambda}\left(s, t_{0}, x\right) v\right\| \\
& \leq N e^{-v(t-s)} e^{-\lambda\left(s-t_{0}\right)}\left\|\Phi_{-\lambda}\left(t, t_{0}, x\right) v\right\| \\
& =N e^{-v(t-s)} e^{\lambda(t-s)}\left\|\Phi\left(t, t_{0}, x\right) v\right\|,
\end{aligned}
$$

for all $(t, s),\left(s, t_{0}\right) \in T$ and all $(x, v) \in Y$. If we consider $v_{3}$ defined as in (34), the second relation in $\left(\mathrm{et}_{3}\right)$ is obtained. Hence, also (iii) in Definition 13 is true and $C$ is $\omega$ trichotomic.

Another characterization for the property of $\omega$ trichotomy is given relative to the dual space $V^{*}$ of the Banach space $V$. To this aim, let us consider three projections families $\left\{P_{k}\right\}_{k \in\{1,2,3\}}$ compatible with $C$ such that the evolution cocycle $\Phi_{1}$ has exponential growth and the evolution cocycle $\Phi_{2}$ has exponential decay.

Theorem 19. A*-strongly measurable skew-evolution semiflow $C=(\varphi, \Phi)$ is $\omega$-trichotomic if the and only if the following statements hold:

(i) there exists $\widetilde{N} \geq 1$ such that

$$
\int_{t_{0}}^{t}\left\|\Phi_{1}\left(t, s, \varphi\left(s, t_{0}, x\right)\right)^{*} v^{*}\right\|^{p} d s \leq \widetilde{N}\left\|P_{1}(x) v^{*}\right\|^{p},
$$

$$
\text { for all } p>1 \text {, all }\left(t, t_{0}\right) \in T \text {, and all }\left(x, v^{*}\right) \in X \times V^{*} ;
$$

(ii) there exists $\bar{N} \geq 1$ such that

$$
\left(\int_{t_{0}}^{t}\left\|\Phi_{2}\left(s, t_{0}, x\right) v\right\|^{p} d s\right)^{1 / p} \leq \bar{N}\left\|\Phi_{2}\left(t, t_{0}, x\right) v\right\|,
$$

for all $p>1$, all $\left(t, t_{0}\right) \in T$, and all $(x, v) \in Y ;$

(iii) there exist $\alpha<0$ and $\widetilde{M} \geq 1$ such that

$$
\int_{t_{0}}^{\infty} e^{\alpha\left(\tau-t_{0}\right)}\left\|\Phi_{3}\left(\tau, t_{0}, x\right) v\right\| d \tau \leq \widetilde{M}\left\|P_{3}(x) v\right\|,
$$

for all $t_{0} \in \mathbb{R}_{+}$and all $(x, v) \in Y$ and there exist $\beta<0$ and $\bar{M} \geq 1$ such that

$$
\int_{t_{0}}^{t} e^{\beta(t-\tau)}\left\|\Phi_{3}\left(\tau, t_{0}, x\right) v\right\| d \tau \leq \bar{M}\left\|\Phi_{3}\left(t, t_{0}, x\right) v\right\|,
$$

$$
\text { for all }\left(t, t_{0}\right) \in T \text { and all }(x, v) \in Y \text {. }
$$

Proof. Necessity. (i) As $C$ is $\omega$-trichotomic, according to Definition 13 , there exists a mapping $\psi: \mathbb{R}_{+} \rightarrow \mathbb{R}_{+}^{*}$, with the property $\bar{\omega}_{\psi}<0$, such that

$$
\begin{array}{r}
\left\|\Phi\left(t, t_{0}, x\right) P_{1}(x) v\right\| \leq \psi\left(t-t_{0}\right)\left\|P_{1}(x) v\right\|, \\
\forall\left(t, t_{0}\right) \in T, \quad(x, v) \in Y .
\end{array}
$$


An equivalent relation is obtained, if we consider Definition 2 $\left(\mathrm{ec}_{2}\right)$, given by

$$
\begin{aligned}
\left\|\Phi_{1}\left(t, t_{0}, x\right) v\right\| & =\left\|\Phi_{1}\left(t, s, \varphi\left(s, t_{0}, x\right)\right) \Phi_{1}\left(s, t_{0}, x\right) v\right\| \\
& \leq \psi(t-s)\left\|\Phi_{1}\left(s, t_{0}, x\right) v\right\|
\end{aligned}
$$

for all $\left(t, t_{0}\right) \in T$ and all $(x, v) \in Y$. According to the properties of function $\psi$, there exist $N \geq 1$ and $\nu>0$ such that the following relations hold:

$$
\begin{aligned}
\int_{t_{0}}^{t} \| & \Phi_{1}\left(t, s, \varphi\left(s, t_{0}, x\right)\right)^{*} v^{*} \|^{p} d s \\
& \leq \int_{t_{0}}^{t} \psi^{p}(t-s)\left\|\Phi_{1}\left(s, s, \varphi\left(s, t_{0}, x\right)\right)^{*} v^{*}\right\|^{p} d s \\
& \leq N^{p} \int_{t_{0}}^{t} e^{-p v(t-s)}\left\|P_{1}(x) v^{*}\right\|^{p} d s \\
& \leq \widetilde{N}\left\|P_{1}(x) v^{*}\right\|^{p},
\end{aligned}
$$

for all $\left(t, t_{0}\right) \in T$ and all $\left(x, v^{*}\right) \in X \times V^{*}$, where we have denoted that $\widetilde{N}=N^{p} / \nu p$.

(ii) According to Definition 13, there exists a mapping $\zeta$ : $\mathbb{R}_{+} \rightarrow \mathbb{R}_{+}^{*}$, with the property $\underline{\omega}_{\zeta}<0$, such that

$$
\begin{array}{r}
\zeta\left(t-t_{0}\right)\left\|P_{2}(x) v\right\| \leq\left\|\Phi_{2}\left(t, t_{0}, x\right) v\right\|, \\
\forall\left(t, t_{0}\right) \in T,(x, v) \in Y .
\end{array}
$$

The property of function $\zeta$ assures the existence of some constants $N \geq 1$ and $v>0$, such that, for all $p>0$, the following relations hold:

$$
\begin{aligned}
\left(\int_{t_{0}}^{t}\left\|\Phi_{2}\left(s, t_{0}, x\right) v\right\|^{p} d s\right)^{1 / p} \leq & N\left\|\Phi_{2}\left(t, t_{0}, x\right) v\right\| \\
& \times\left(\int_{t_{0}}^{t} e^{-v p(t-s)} d s\right)^{1 / p} \\
\leq & \bar{N}\left\|\Phi_{2}\left(t, t_{0}, x\right) v\right\|
\end{aligned}
$$

for all $\left(t, t_{0}\right) \in T$ and all $(x, v) \in Y$, where we have denoted that $\bar{N}=N /(v p)^{1 / p}$.

(iii) Both relations are obtained by a similar proof as in (i) and (ii), according to Theorem 18.

Sufficiency. (i) As the evolution cocycle $\Phi_{1}$ has exponential growth, there exist $M \geq 1$ and $\rho>0$ such that

$$
\begin{array}{r}
\left\|\Phi_{1}\left(t, t_{0}, x\right) v\right\| \leq M e^{\rho(t-s)}\left\|\Phi_{1}\left(s, t_{0}, x\right) v\right\|, \\
\forall(t, s),\left(s, t_{0}\right) \in T, \quad(x, v) \in Y .
\end{array}
$$

Let $t \geq t_{0}+1$. We consider that

$$
\int_{t_{0}}^{t_{0}+1} e^{-\rho\left(s-t_{0}\right)} d s=\int_{0}^{1} e^{-\rho \tau} d \tau=\frac{1-e^{-\rho}}{\rho}=c>0 .
$$

For every $p>1$ there exists $q>1$ such that $1 / p+1 / q=1$. We obtain

$$
\begin{aligned}
& c\left|\left\langle v^{*}, \Phi_{1}\left(t, t_{0}, x\right) v\right\rangle\right| \\
& \leq \int_{t_{0}}^{t_{0}+1} e^{-\rho\left(s-t_{0}\right)}\left|\left\langle v^{*}, \Phi_{1}\left(t, t_{0}, x\right) v\right\rangle\right| d s \\
& \leq \int_{t_{0}}^{t_{0}+1} e^{-\rho\left(s-t_{0}\right)}\left\|\Phi_{1}\left(t, s, \varphi\left(s, t_{0}, x\right)\right)^{*} v^{*}\right\| \\
& \quad \times\left\|\Phi_{1}\left(s, t_{0}, x\right) v\right\| d s \\
& \leq M\|v\| \int_{t_{0}}^{t_{0}+1}\left\|\Phi_{1}\left(t, s, \varphi\left(s, t_{0}, x\right)\right)^{*} v^{*}\right\| d s \\
& \leq\left(\int_{t_{0}}^{t_{0}+1}\left\|\Phi_{1}\left(t, s, \varphi\left(s, t_{0}, x\right)\right)^{*} v^{*}\right\|^{p} d s\right)^{1 / p} \\
& \quad \times\left(\int_{t_{0}}^{t_{0}+1} M^{q}\left\|P_{1}(x) v\right\|^{q} d s\right)^{1 / q} \\
& \leq M\|v\|\left(\int_{t_{0}}^{t}\left\|\Phi_{1}\left(t, s, \varphi\left(s, t_{0}, x\right)\right)^{*} v^{*}\right\|^{p} d s\right)^{1 / p} \\
& \leq M \widetilde{N}\left\|P_{1}(x) v\right\|\left\|P_{1}(x) v^{*}\right\| .
\end{aligned}
$$

Hence, we have that

$$
\begin{array}{r}
\left\|\Phi_{1}\left(t, s, \varphi\left(s, t_{0}, x\right)\right) v\right\| \leq \frac{M \widetilde{N}}{c}\left\|P_{1}(x) v\right\|, \\
\forall t \geq t_{0}+1, \forall(x, v) \in Y .
\end{array}
$$

For $t \in\left[t_{0}, t_{0}+1\right]$ we obtain

$$
\left\|\Phi_{1}\left(t, s, \varphi\left(s, t_{0}, x\right)\right) v\right\| \leq M e^{\rho}\left\|P_{1}(x) v\right\|, \quad \forall(x, v) \in Y,
$$

which implies that

$$
\begin{array}{r}
\left\|\Phi_{1}\left(t, s, \varphi\left(s, t_{0}, x\right)\right) v\right\| \leq M_{1}\left\|P_{1}(x) v\right\|, \\
\forall\left(t, t_{0}\right) \in T, \quad(x, v) \in Y,
\end{array}
$$

where we have denoted

$$
M_{1}=M\left(e^{\rho}+\frac{\widetilde{N}}{c}\right) .
$$

Further, the following relations hold:

$$
\begin{aligned}
\mid\left\langle v^{*},\right. & \left.\Phi_{1}\left(t, t_{0}, x\right) v\right\rangle \mid \\
& =\left|\left\langle v^{*}, \Phi_{1}\left(t, s, \varphi\left(s, t_{0}, x\right)\right) \Phi_{1}\left(s, t_{0}, x\right) v\right\rangle\right| \\
& =\left|\left\langle\Phi_{1}\left(t, s, \varphi\left(s, t_{0}, x\right)\right)^{*} v^{*}, \Phi_{1}\left(s, t_{0}, x\right) v\right\rangle\right| \\
& \leq M_{1}\left\|P_{1}(x) v\right\|\left\|\Phi_{1}\left(t, s, \varphi\left(s, t_{0}, x\right)\right)^{*} v^{*}\right\| .
\end{aligned}
$$


By integrating on $\left[t_{0}, t\right]$, it follows that

$$
\begin{aligned}
& \left(t-t_{0}\right)\left|\left\langle v^{*}, \Phi_{1}\left(t, t_{0}, x\right) v\right\rangle\right| \\
& \quad \leq M_{1}\left\|P_{1}(x) v\right\| \int_{t_{0}}^{t}\left\|\Phi_{1}\left(t, s, \varphi\left(s, t_{0}, x\right)\right)^{*} v^{*}\right\| d s \\
& \leq M_{1} \widetilde{N}\left\|P_{1}(x) v\right\|\left\|P_{1}(x) v^{*}\right\|,
\end{aligned}
$$

and further

$$
\left(t-t_{0}\right)\left\|\Phi_{1}\left(t, t_{0}, x\right) v\right\| \leq M_{1} \widetilde{N}\left\|P_{1}(x) v\right\|,
$$

which implies that

$$
\begin{array}{r}
\left\|\Phi_{1}\left(t, t_{0}, x\right) v\right\| \leq \psi\left(t-t_{0}\right)\left\|P_{1}(x) v\right\|, \\
\forall\left(t, t_{0}\right) \in T, \quad(x, v) \in Y,
\end{array}
$$

where we have considered $\psi(u)=M_{1}(\widetilde{N}+1) / u$, which satisfies $\bar{\omega}_{\psi}<0$. Statement $\left(t_{1}\right)$ of Definition 13 is hence obtained.

(ii) Let $\left(t, t_{0}\right) \in T$. Let us denote that

$$
F(t)=\int_{t_{0}}^{t}\left\|\Phi_{2}\left(s, t_{0}, x\right) v\right\|^{p} d s .
$$

According to the fact that $F(t) \leq \bar{N}^{p} F^{\prime}(t)$, for all $t \geq 0$, we obtain, for all $(x, v) \in Y$,

$$
F\left(t_{0}+1\right) e^{\left(t-t_{0}-1\right) / \bar{N}^{p}} \leq F(t) \leq \bar{N}^{p}\left\|\Phi_{2}\left(t, t_{0}, x\right) v\right\|^{p} .
$$

As the evolution cocycle $\Phi_{2}$ has exponential decay, there exist some constants $M \geq 1$ and $\rho>0$ such that

$$
\begin{array}{r}
\left\|\Phi_{2}\left(s, t_{0}, x\right) v\right\| \leq M e^{\rho(t-s)}\left\|\Phi_{2}\left(t, t_{0}, x\right) v\right\|, \\
\forall(t, s),\left(s, t_{0}\right) \in T, \quad(x, v) \in Y .
\end{array}
$$

Let $s \in\left[t_{0}, t_{0}+1\right)$. We obtain

$$
\left\|P_{2}(x) v\right\|^{p} \leq M^{p} e^{p \rho(t-s)} \int_{t_{0}}^{t_{0}+1}\left\|\Phi_{2}\left(s, t_{0}, x\right) v\right\|^{p} d s
$$

and further

$$
M^{-p} e^{-p \rho(t-s)}\left\|P_{2}(x) v\right\|^{p} \leq F\left(t_{0}+1\right) .
$$

For $t \geq t_{0}+1$, we obtain

$$
\frac{1}{M^{p} e^{\rho p}} e^{-1 / \bar{N}^{p}} e^{\left(t-t_{0}\right) / \bar{N}^{p}}\|v\|^{p} \leq \bar{N}^{p}\left\|\Phi_{2}\left(t, t_{0}, x\right) v\right\|^{p},
$$

and, if $t \in\left[t_{0}, t_{0}+1\right]$,

$$
\left\|P_{2}(x) v\right\| \leq M e^{\rho} e^{1 / p^{\bar{N}^{p}}} e^{\left(-1 / p^{\bar{N}^{p}}\right)\left(t-t_{0}\right)}\left\|\Phi_{2}\left(t, t_{0}, x\right) v\right\|,
$$

for all $(x, v) \in Y$. Hence,

$$
\begin{array}{r}
\zeta\left(t-t_{0}\right)\left\|P_{2}(x) v\right\| \leq\left\|\Phi_{2}\left(t, t_{0}, x\right) v\right\|, \\
\forall\left(t, t_{0}\right) \in T,(x, v) \in Y,
\end{array}
$$

where we have considered $\zeta: \mathbb{R}_{+} \rightarrow \mathbb{R}_{+}^{*}$ defined by

$$
\zeta(u)=\frac{1}{\bar{N} M e^{\rho}} e^{-1 / p^{\bar{N}^{p}}} e^{\left(1 / p^{\bar{N}^{p}}\right) u},
$$

with the property $\underline{\omega}_{\zeta}>0$. Statement $\left(t_{2}\right)$ of Definition 13 is proved.

(iii) A similar proof for the $\alpha$-shifted evolution cocycle $\Phi_{\alpha}^{3}$ in (i) and for the $\beta$-shifted evolution cocycle $\Phi_{\beta}^{3}$ in (ii) leads to the existence of two functions $\chi_{1}, \chi_{2}: \mathbb{R}_{+} \rightarrow \mathbb{R}_{+}^{*}$, with the properties $\bar{\omega}_{\chi_{1}}<0$ and $\underline{\omega}_{\chi_{2}}>0$, such that

$$
\begin{array}{r}
\left\|\Phi_{\alpha}^{3}\left(t, t_{0}, x\right) v\right\| \leq \chi_{1}\left(t-t_{0}\right)\left\|P_{3}(x) v\right\|, \\
\forall\left(t, t_{0}\right) \in T, \quad(x, v) \in Y, \\
\chi_{2}\left(t-t_{0}\right)\left\|P_{3}(x) v\right\| \leq\left\|\Phi_{\beta}^{3}\left(t, t_{0}, x\right) v\right\|, \\
\forall\left(t, t_{0}\right) \in T,(x, v) \in Y .
\end{array}
$$

If we consider the definition of the shifted evolution cocycle, the previous relations are equivalent with

$$
\begin{array}{r}
\left\|\Phi_{3}\left(t, t_{0}, x\right) v\right\| \leq e^{-\alpha\left(t-t_{0}\right)} \chi_{1}\left(t-t_{0}\right)\left\|P_{3}(x) v\right\|, \\
\forall\left(t, t_{0}\right) \in T,(x, v) \in Y, \\
e^{-\beta\left(t-t_{0}\right)} \chi_{2}\left(t-t_{0}\right)\left\|P_{3}(x) v\right\| \leq\left\|\Phi_{3}\left(t, t_{0}, x\right) v\right\|, \\
\forall\left(t, t_{0}\right) \in T,(x, v) \in Y .
\end{array}
$$

We define $\tilde{\chi}, \bar{\chi}: \mathbb{R}_{+} \rightarrow \mathbb{R}_{+}^{*}$ by $\tilde{\chi}(u)=e^{-\alpha u} \chi_{1}(u)$ and $\bar{\chi}(u)=$ $e^{-\beta u} \chi_{2}(u)$, which have the properties $\bar{\omega}_{\tilde{\chi}}<\infty$ and $\underline{\omega}_{\bar{\chi}}<0$. Hence, property $\left(t_{3}\right)$ of Definition 13 is obtained.

Remark 20. The property described by relation (36) is also called $*$-strong integral stability for a skew-evolution semiflow. Relation (36) is a characterization of Barbashin type in the strong topology, and relations (37), (38), and (39) are characterizations of Datko type for the asymptotic properties of skew-evolution semiflows involved in the definition of $\omega$ trichotomy.

\section{Acknowledgments}

This paper was conceived during the visit at the Institute of Mathematics of Bordeaux, France. The author wishes to express her profound and respectful gratitude to Professor Bernard Chevreau and Professor Mihail Megan. Also, the author gratefully acknowledges helpful comments and suggestions from the referees.

\section{References}

[1] M. Megan and C. Stoica, "Exponential instability of skew-evolution semi ows in Banach spaces," Studia Universitatis "BabesBolyai" Mathematica, vol. 53, no. 1, pp. 17-24, 2008.

[2] A. J. G. Bento and C. M. Silva, "Nonuniform dichotomic behavior: Lipschitz invariant manifolds for ODEs," http://arxiv .org/abs/1210.7740. 
[3] P. V. Hai, "Continuous and discrete characterizations for the uniform exponential stability of linear skew-evolution semiflows," Nonlinear Analysis, Theory, Methods and Applications, vol. 72, no. 12, pp. 4390-4396, 2010.

[4] P. V. Hai, "Discrete and continuous versions of Barbashintype theorem of linear skew-evolution semiflows," Applicable Analysis, vol. 90, no. 12, pp. 1897-1907, 2011.

[5] M. Megan and C. Stoica, "Concepts of dichotomy for skewevolution semiflows in banach spaces," Annals of the Academy of Romanian Scientists, vol. 2, no. 2, pp. 125-140, 2010.

[6] C. Stoica and M. Megan, "On uniform exponential stability for skew-evolution semiflows on Banach spaces," Nonlinear Analysis, Theory, Methods and Applications, vol. 72, no. 3-4, pp. 1305-1313, 2010.

[7] J. Appell, V. Lakshmikantham, N. V. Minh, and P. P. Zabreiko, "A general model of evolutionary processes. Exponential dichotomy-I," Nonlinear Analysis, vol. 21, no. 3, pp. 207-218, 1993.

[8] A. L. Sasu and B. Sasu, "Integral equations, dichotomy of evolution families on the half-line and applications," Integral Equations and Operator Theory, vol. 66, no. 1, pp. 113-140, 2010.

[9] S. Elaydi and O. Hajek, "Exponential trichotomy of differential systems," Journal of Mathematical Analysis and Applications, vol. 129, no. 2, pp. 362-374, 1988.

[10] M. Megan and C. Stoica, "On uniform exponential trichotomy of evolution operators in banach spaces," Integral Equations and Operator Theory, vol. 60, no. 4, pp. 499-506, 2008.

[11] R. J. Sacker and G. R. Sell, "Existence of dichotomies and invariant splittings for linear differential systems, III," Journal of Differential Equations, vol. 22, no. 2, pp. 497-522, 1976. 


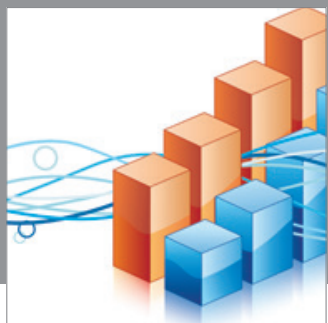

Advances in

Operations Research

mansans

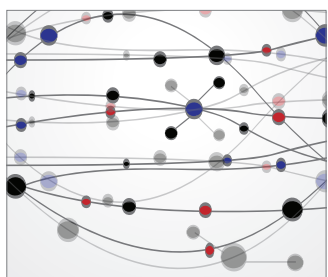

The Scientific World Journal
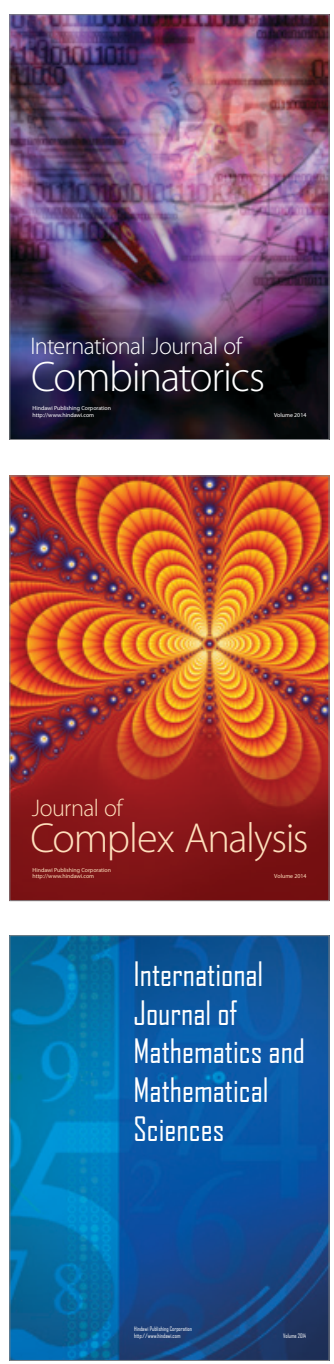
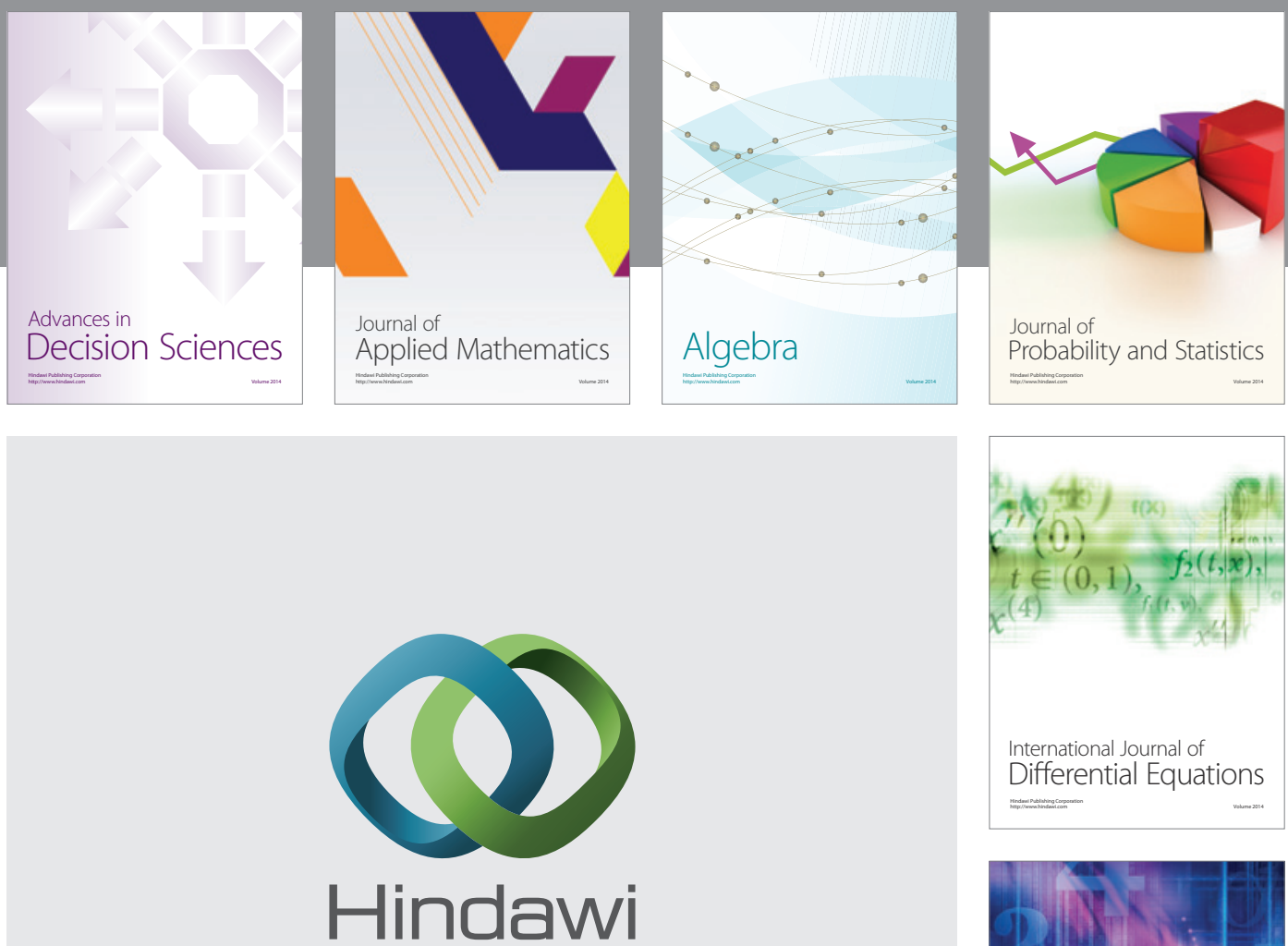

Submit your manuscripts at http://www.hindawi.com
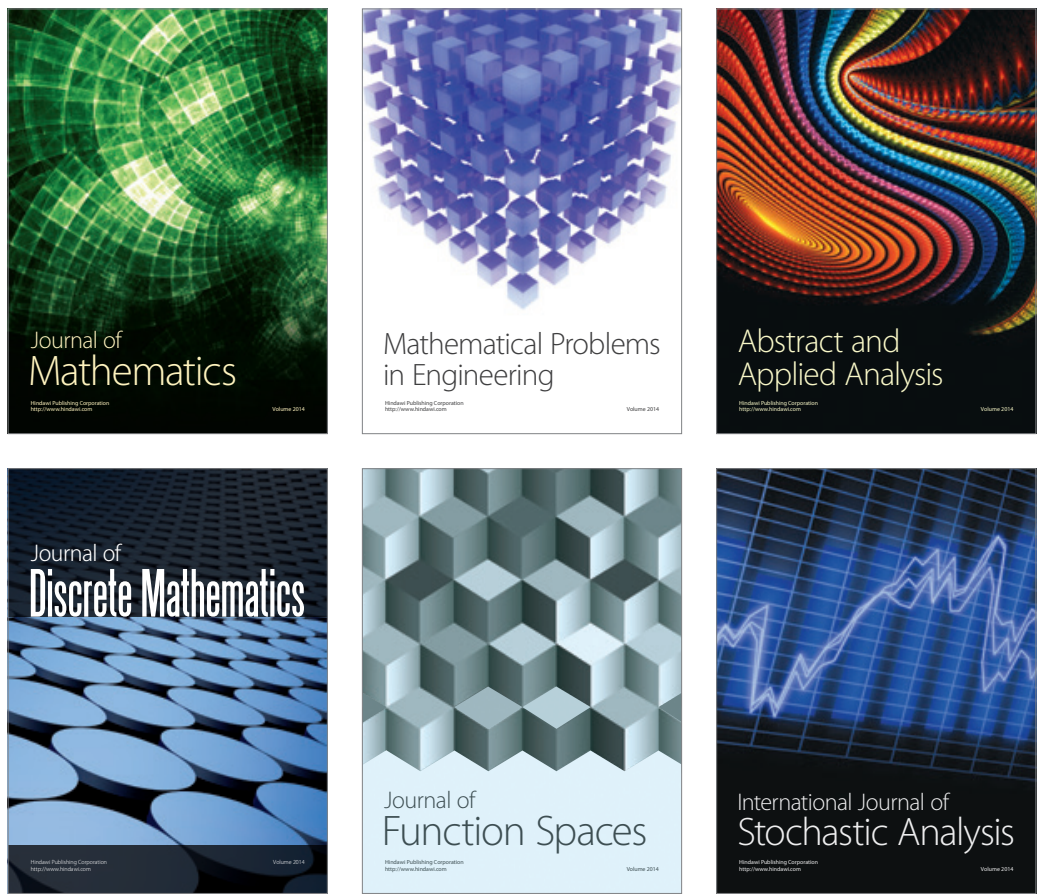

Journal of

Function Spaces

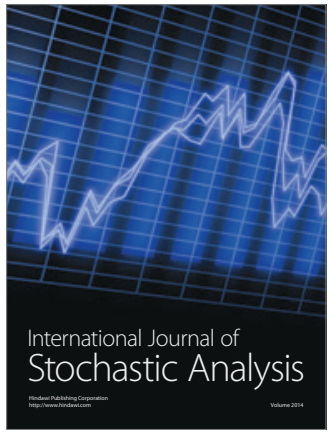

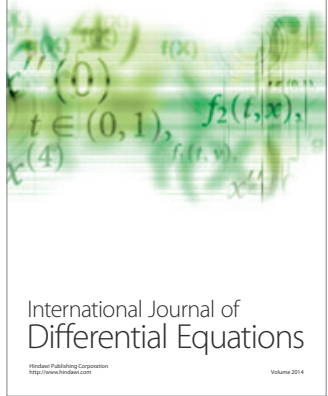
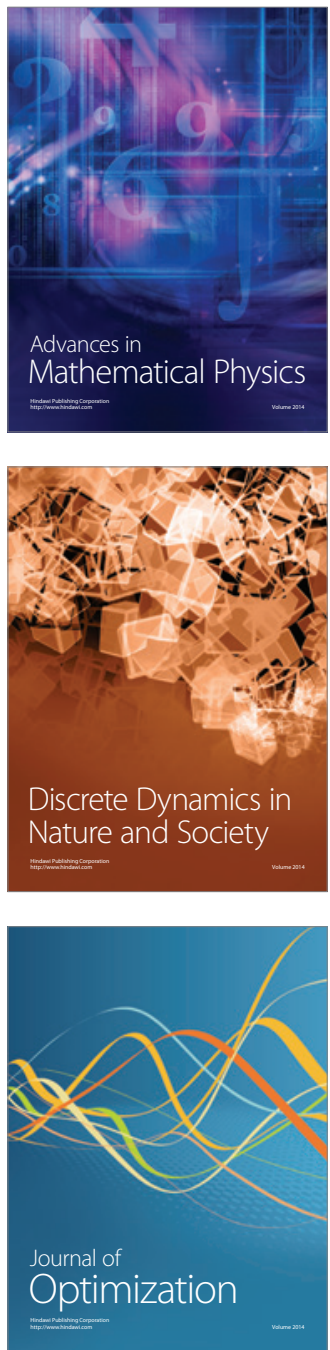